\title{
Situación de la atención primaria de salud mental en servicios públicos de El Alto, La Paz, Bolivia
}

\author{
Carmen Camacho-Arce, ${ }^{1}$ Dora Caballero-Baldivieso ${ }^{2}$ \\ y Francis Venegas-Arzabe ${ }^{3}$
}

Forma de citar Camacho-Arce C, Caballero-Baldivieso D, Venegas-Arzabe F. Situación de la atención primaria de salud mental en servicios públicos de El Alto, La Paz, Bolivia. Rev Panam Salud Publica. 2009;25(6):511-7.

RESUMEN Objetivos. Caracterizar los trastornos de salud mental o conductuales (TSMC) atendidos en una red de salud boliviana y conocer la percepción de profesionales sanitarios, representantes de la comunidad y responsables de brindar los servicios a fin de identificar los elementos necesarios para mejorar la respuesta a estos problemas.

Método. Se realizó un estudio transversal descriptivo entre enero y diciembre de 2005 en los 15 centros públicos de salud de los distritos 2 y 3 pertenecientes a la Red Corea, una de las cuatro redes urbanas del municipio de El Alto, provincia de Murillo, departamento de La Paz, Bolivia. Se utilizó la metodología de investigación y acción participativa mediante entrevistas, sesiones en grupos focales, análisis de los cuadernos de registros de consultas externas e historias clínicas y la observación participante.

Resultados. Solo 0,05\% de las consultas en los servicios de primer nivel correspondian a TSMC. Entre las dificultades percibidas por los médicos para la atención de esos pacientes están la falta de un centro de referencia especializado (60,0\%), la falta de tiempo $(40,0 \%)$, la ausencia de personal especializado $(33,3 \%)$, la carencia de medicamentos $(20,0 \%)$ y la insuficiente capacitación del personal (16,6\%). Los programas departamentales y nacionales tienen limitaciones para la capacitación de los recursos humanos, no existen normas de atención y el sistema de información no registra variables sobre TSMC. Las personas de la comunidad identificaron adecuadamente los problemas de salud mental y las necesidades de atención que a la fecha no tienen respuesta de los servicios.

Conclusiones. Se deben fortalecer la formación y la capacitación del personal de los servicios de atención primaria de salud para lograr una adecuada atención de los trastornos de salud mental. Se debe mejorar la gestión de los servicios y el registro de los trastornos de comportamiento y de salud mental. También se recomienda integrar la participación comunitaria al modelo de abordaje de la salud mental dentro del marco de la atención primaria de salud.

Palabras clave Salud mental, servicios de salud, atención primaria de salud, Bolivia.

1 Instituto de Investigación de Psicología, Universidad Mayor de San Andrés, La Paz, Bolivia. La correspondencia se debe dirigir a Carmen Camacho Arce, Calle General Trigo No. 264, Tarija, Bolivia. Correo electrónico: ccamachot@gmail.com

2 Organización Panamericana de la Salud, La Paz, Bolivia.

3 Programa de Salud Mental, Servicio Departamental de Salud, Prefectura de La Paz, Bolivia.
Según el informe de la Organización Mundial de la Salud (OMS) sobre la salud mundial en 2001, una de cada cuatro familias tiene al menos un miembro afectado por trastornos de salud mental o conductuales (TSMC) (1). Muchas de esas personas y sus familias sufren el rechazo de la comunidad e incluso de sus parientes, lo que les dificulta participar en actividades y redes sociales (2). Según el informe de la OMS, las relaciones sexuales sin protección y el consumo de tabaco y alcohol — factores directamente relacionados con los TSMC - se encuentran entre los 10 principales factores de riesgo de enfermedad; sin embargo, 
otros de esos factores - como el sobrepeso, la hipertensión arterial y la colesterolemia elevada- pueden también estar asociados, si bien indirectamente, con la salud mental y las conductas de riesgo $(3,4)$.

Los TSMC pueden tener diversas características y manifestaciones, tanto por su nivel de gravedad como por su duración. Los más frecuentes en la Región de las Américas son la depresión, los trastornos por consumo de sustancias psicoactivas, la esquizofrenia, la epilepsia, la enfermedad de Alzheimer, el retraso mental, los trastornos de la infancia y la adolescencia, la comorbilidad con otras enfermedades somáticas (como la depresión asociada con el cáncer) y el suicidio (1).

Según datos del Ministerio de Salud y Deportes de Bolivia, 7\% de la población infantil atendida en las consultas externas de los centros asistenciales adscritos a la Caja Nacional de Salud, perteneciente al subsistema de seguridad social de ese país, presenta trastornos emocionales o conductuales, tales como enuresis, depresión y retraso mental $(5,6)$. Sin embargo, a pesar de la elevada incidencia de trastornos mentales que reflejan estos datos, en Bolivia no se ha realizado un esfuerzo sostenido para mejorar la atención de estos problemas de salud. Entre otras deficiencias, en el país no existe un plan nacional de salud mental, el Sistema Nacional de Información en Salud no registra los índices de prevalencia e incidencia de los TSMC y los registros de atención en los diferentes centros especializados no son comparables entre sí (7).

Debido a la importancia de contar con información validada sobre la atención y el control de los TSMC en Bolivia, tanto desde la perspectiva de la atención primaria de salud (APS) como de la percepción de la comunidad, se realizó la presente investigación. Sus objetivos fueron caracterizar los TSMC atendidos en una red de salud y conocer la percepción de profesionales sanitarios, representantes de la comunidad y responsables de brindar los servicios a fin de identificar los elementos necesarios para mejorar la respuesta a estos problemas.

\section{MATERIALES Y MÉTODOS}

Se realizó un estudio transversal descriptivo en centros de APS en los distritos 2 y 3 del municipio de El Alto, pro- vincia de Murillo, departamento de La Paz, Bolivia.

El Alto cuenta con 69 servicios de salud -entre públicos (45\%), privados (50\%) y de la seguridad social (5\%)para atender una población de 649958 habitantes (8). La tasa de alfabetismo en El Alto es de 92,0\% (97,6\% en hombres y $86,8 \%$ en mujeres); $49,3 \%$ de la población se ubica en el nivel de pobreza moderada, $25,0 \%$ en el umbral de pobreza, $17,0 \%$ en el nivel de indigencia y solo $7,4 \%$ tienen sus necesidades básicas satisfechas. En general, 51,0\% de los partos se realizan en instituciones de salud y la cobertura de vacunación contra el sarampión en niños y niñas de 12 a 23 meses de edad es de 53,0\%. La mortalidad infantil se estima en 64,1 por 1000 nacidos vivos, similar a la informada para todo el departamento de La Paz (9).

El estudio se realizó de enero a diciembre de 2005 en los 15 centros públicos de salud de los distritos 2 y 3 pertenecientes a la Red Corea, una de las cuatro redes urbanas del municipio que en ese momento concentraba $48,4 \%$ de los servicios públicos y $24,0 \%$ del total de los servicios de salud. Estos servicios atendían a una población de 224772 habitantes $(34,6 \%$ de la población total del municipio) $(10,11)$. De los 15 servicios seleccionados, 14 eran de primer nivel y 1 de segundo nivel.

Los criterios utilizados para seleccionar esta red fueron: atendía una población urbana concentrada, tenía servicios públicos de salud tanto ambulatorios como hospitalarios con al menos un servicio de salud mental y contaba con la participación comunitaria mediante el Directorio Local de Salud. ${ }^{4}$

Se utilizó la metodología de investigación y acción participativa (12) con técnicas cuantitativas y cualitativas. En cada uno de los 15 centros seleccionados se realizaron dos visitas de observación a consultas médicas y se aplicó una encuesta semiestructurada a un médico de consulta externa. Además se hicieron entrevistas en profundidad a dos autoridades sanitarias locales y a dos grupos focales conformados por cinco líderes comunitarios y cinco usuarios de los servicios que forman parte de la Red Social

\footnotetext{
4 El Directorio Local de Salud es una instancia de gestión compartida en salud conformada por un representante del Servicio Departamental de Salud, un representante del gobierno municipal y un representante de la comunidad organizada.
}

de Salud. ${ }^{5}$ Un médico especialista en salud pública analizó los cuadernos de registro de las consultas realizadas y las historias clínicas de las consultas externas, correspondientes al segundo semestre de 2004, en busca de información sobre casos de trastornos depresivos, consumo de sustancias psicoactivas, esquizofrenia, epilepsia, Alzheimer, retraso mental, trastornos de la infancia y la adolescencia, comorbilidad y suicidio. Se construyeron tablas ordinales a partir de las frecuencias porcentuales acumuladas no excluyentes de los casos detectados.

Se realizó el diagnóstico de la situación de la atención en salud mental mediante técnicas cuantitativas (a partir de indicadores y variables codificadas de las entrevistas semiestructuradas) y análisis cualitativo de las observaciones y entrevistas en profundidad.

\section{RESULTADOS}

En los 15 centros de la red estudiada laboraban 107 profesionales, técnicos y trabajadores administrativos. De ellos, 48 eran profesionales: 43 eran médicos (1,4 × 10000 habitantes), 8 eran odontólogos, 7 eran licenciados en enfermería y uno era trabajador social. Además, se contaba con un técnico estadístico, 37 auxiliares de enfermería y 21 personas que ocupaban cargos administrativos. El único centro de referencia de la red es el Hospital Corea, de segundo nivel, que concentra a médicos especialistas $(4,0 \%)$ y donde dos psicólogos hacían pasantías; ningún psiquiatra integraba el plantel.

En ninguno de los centros estudiados se encontraron protocolos que regularan la atención de los TSMC en los servicios de APS.

\section{Perfil de la demanda}

Según la revisión documental, durante el segundo semestre de 2004 se realizó un total de 28791 primeras consultas en los 15 servicios de salud, de ellas solo 15 $(0,05 \%)$ correspondían a TSMC. Sin embargo, 67,0\% de los casos estaban relacionados de manera secundaria con hechos de violencia doméstica, 13,0\% con epilepsia y $7,0 \%$ con retraso mental.

\footnotetext{
5 La Red Social de Salud es una organización integrada por personas e instituciones locales, organizadas en forma paralela a la red de servicios para facilitar el acceso de la comunidad a los centros de salud, la atención a las mujeres y los niños, la gestión de los recursos y la evaluación de la calidad de la atención sanitaria.
} 


\section{Consulta externa y recursos humanos}

De los 30 médicos visitados durante sus consultas externas, solo $42,9 \%$ interrogó y registró hábitos, antecedentes personales y familiares. Ninguno profundizó en temas de salud mental durante el interrogatorio. La mayoría de los médi$\cos (71,8 \%)$ dio indicaciones a los pacientes sobre el tratamiento; solo $28,2 \%$ corroboró la comprensión de los pacientes sobre las indicaciones realizadas a pesar de haber utilizado un lenguaje técnico. En las visitas observadas no se recetó ningún medicamento tranquilizante, ansiolítico o de uso psiquiátrico.

El tiempo promedio de duración de las consultas fue de aproximadamente 15 minutos. Los servicios visitados tenían una elevada demanda de pacientes ( 25 consultas por médico en una jornada de 6 horas, como promedio), lo que puede explicar la corta duración de las consultas. Además de atender a los pacientes, los médicos debían llenar los formularios de notificación obligatoria por infecciones respiratorias agudas y enfermedades diarreicas agudas, así como los informes del Sistema Nacional de Información en Salud y del seguro universal materno-infantil.

Según la percepción de los médicos, el motivo más frecuente de consulta fueron las infecciones respiratorias agudas $\mathrm{y}$ las enfermedades diarreicas agudas (cuadro 1).

\section{Percepción de los médicos sobre los TSMC}

De los médicos entrevistados, 47,0\% manifestó que en los servicios de APS se habían presentado casos relacionados con la salud mental, entre ellos alcoholismo, adicción a las drogas, ansiedad, violencia intrafamiliar, violaciones, epilepsia y esquizofrenia.

Según la percepción de los médicos, las dificultades para la atención de los TSMC eran no contar con un centro de referencia para esos pacientes $(60,0 \%)$, falta de tiempo $(40,0 \%)$, no contar con personal especializado $(33,3 \%)$, falta de cooperación del paciente $(27,0 \%)$, carencia de medicamentos $(20,0 \%)$, insuficiente capacitación del personal $(16,6 \%)$ y falta de un sistema de contrarreferencia (los pacientes no se remitían de vuelta al servicio de atención primaria una vez que terminaban su tratamiento en las instituciones de salud de segundo o tercer nivel a los que se remitían) $(13,3 \%)$. De los médicos entrevistados, $43,3 \%$ dijo que no había casos de TSMC en el servicio, de los cuales 7,0\% afirmó que esos pacientes deben atenderse en servicios de tercer nivel y 7,6\% dijo que no había dificultades porque estos pacientes se derivaban al Hospital Corea.

Entre las causas de la falta de atención de los TSMC en los servicios de APS identificadas por los médicos se encontraban: no contar con apoyo logístico y médicos especialistas para tratar los casos de salud mental $(68,1 \%)$, no tener un centro de tercer nivel a donde remitirlos $(53,7 \%)$ y no contar con posibilidades para realizar el seguimiento de esos pacientes $(40,0 \%)$, ya que recién se estaba poniendo en funcionamiento el sistema de contrarreferencia en la red. Una parte de los médicos (20,0\%) manifestó que los pacientes no acudían a las consultas a las que eran referidos.

De los médicos consultados, 47,0\% afirmó que en su servicio había casos de

CUADRO 1. Percepción de los médicos sobre las causas más frecuentes de consulta en la red urbana de servicios públicos de salud de los distritos 2 y 3 , municipio de El Alto, La Paz, Bolivia, 2004

\begin{tabular}{lcc}
\hline \multicolumn{1}{c}{$\begin{array}{c}\text { Percepción sobre causas } \\
\text { frecuentes de consulta }\end{array}$} & $\begin{array}{c}\text { No. de } \\
\text { respuestas }\end{array}$ & $\begin{array}{c}\text { Orden de } \\
\text { frecuencia }\end{array}$ \\
\hline $\begin{array}{l}\text { Infecciones respiratorias agudas, enfermedades } \\
\text { diarreicas agudas }\end{array}$ & 30 & 1 \\
Desnutrición & 29 & 2 \\
Dermatosis & 27 & 3 \\
Accidentes & 24 & 4 \\
Parasitosis intestinal & 20 & 5 \\
Control prenatal & 19 & 6 \\
Infecciones urinarias & 17 & 7 \\
Enfermedades de transmisión sexual & 16 & 8 \\
Planificación familiar & 14 & 9 \\
Otras (intoxicaciones, erupciones alérgicas, abortos) & 11 & 10 \\
\hline
\end{tabular}

TSMC y consideró como factores de riesgo la mayor edad y el sexo femenino $(28,0 \%)$, la inestabilidad familiar y afectiva $(17,0 \%)$, la falta de información sobre la salud $(17,0 \%)$, el desempleo $(11,0 \%)$, el consumo de alcohol y drogas $(7,0 \%)$, la pobreza $(7,0 \%)$ y las consultas tardías $(7,0 \%)$; por su parte, $34,0 \%$ de esos médicos consideró que los casos de TSMC han sido poco frecuentes.

Solo en $10(66,6 \%)$ de los servicios de la red había medicamentos psicotrópicos disponibles, entre ellos el Hospital Corea, de segundo nivel. Los medicamentos disponibles encontrados fueron: diazepam, atropina, alprazolam y anticonvulsionantes (estos últimos se encontraron solamente en un servicio).

\section{Necesidades para mejorar los servicios en salud mental}

En opinión de los médicos entrevistados, para mejorar los servicios de atención en salud mental es necesario desarrollar algunas líneas de trabajo, entre ellas emprender acciones de capacitación, establecer un servicio de referencia, mejorar la coordinación entre los servicios, aumentar la participación de los familiares y contar con un psicólogo (figura 1). Una parte de los médicos destacaron la importancia de contar con un modelo de atención apropiado, ambientes exclusivos para la atención de los casos de TSMC e involucrar a la red social.

\section{Percepción de autoridades sanitarias y líderes comunitarios}

Los resultados de las entrevistas en profundidad a autoridades sanitarias y grupos focales comunitarios sobre las situaciones de riesgo de los TSMC más frecuentes se sintetizan en el cuadro 2 .

Las observaciones de los líderes de organizaciones comunitarias sobre los TSMC más frecuentes, sus factores de riesgo y las barreras para lograr una mejor atención estuvieron relacionadas fundamentalmente con aspectos socioculturales, como el machismo, la violencia, la inequidad de género, la necesidad de capacitarse y el cambio en las funciones de las instituciones y organizaciones sociales para que coordinen mejor su trabajo y tomen en cuenta a la comunidad (cuadro 2).

Por su parte, las opiniones predominantes en las autoridades de salud se concentraron en los factores que genera- 
FIGURA 1. Percepción de los médicos sobre las necesidades para mejorar los servicios de salud mental y conductuales en la red urbana de servicios públicos de salud de los distritos 2 y 3 , municipio de El Alto, La Paz, Bolivia, 2004

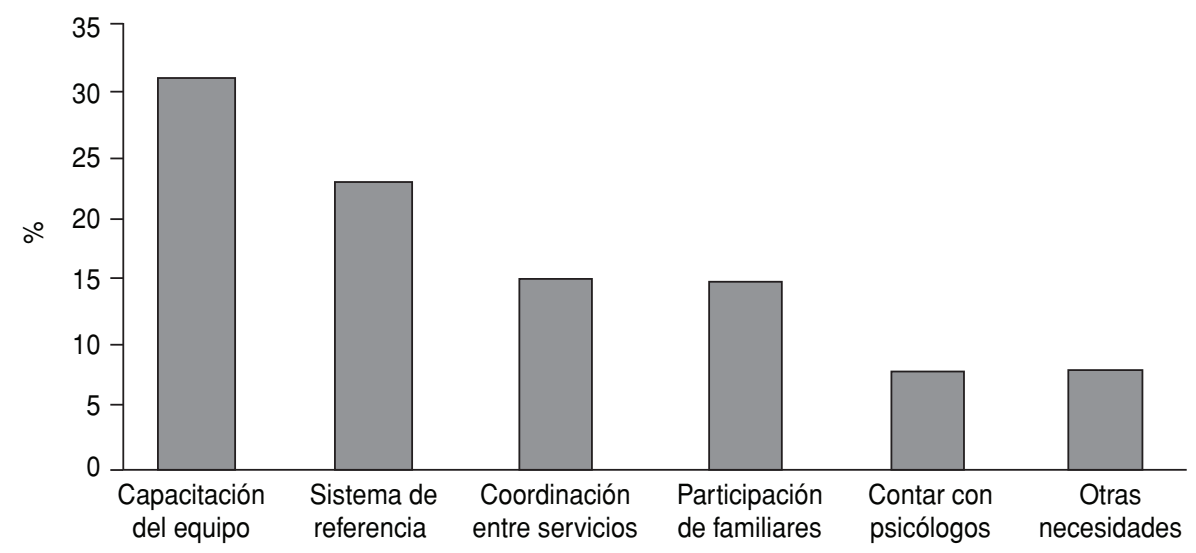

ban problemas de salud mental, como el difícil acceso a fuentes de trabajo, la inequidad de género y la inseguridad (cuadro 2).

Opinión de los usuarios y las usuarias de los servicios de salud

De los 45 usuarios y usuarias que integraban los grupos focales, 93,3\% eran mujeres. Esto se debió a la mayor oferta de servicios dirigidos a las mujeres en edad reproductiva y a los menores de 5 años, ambos cubiertos por el seguro universal materno-infantil.

La mayor parte $(71,2 \%)$ de los usuarios entrevistados (uno por cada centro de salud, independientemente del motivo de la consulta) dijo que el médico no les informó su diagnóstico. A la pregunta sobre su percepción acerca de su estado de salud, 27,0\% afirmó estar preocupado y $20,0 \%$ que no les permitía trabajar; el restante 53,0\% dijo que no sabía. Cuando se les preguntó cuál era su fuente de información sobre cuestiones de salud, 53,3\% dijo que con las amistades, $26,7 \%$ con el servicio de salud y $20,0 \%$ con familiares; el hospital de referencia no se identificó como una fuente de información.

\section{DISCUSIÓN}

En los servicios estudiados no se ha registrado una demanda sistemática de atención por TSMC, ya sea porque la población no acude a los centros de salud por problemas relacionados con este tipo de trastornos o porque no tiene conciencia del posible carácter de salud mental de algunas dolencias psicosomáticas. Por otra parte, el interrogatorio de los médi$\cos$ no profundizaba en las causas que llevaban al paciente a buscar ayuda médica, lo que puede haber impedido identificar una gran parte de esos trastornos. En consecuencia, la información sobre los TSMC en las historias clínicas y los cuadernos de registro es prácticamente nula y no permite identificar los factores de riesgo asociados con los TSMC más frecuentes.

Según la percepción de los médicos, los principales factores de riesgo de TSMC fueron la edad (jóvenes de 11 a 19 años y personas mayores de 60 años) y el sexo femenino, aunque también mencionaron otros factores, como la violencia familiar. Según los miembros de la comunidad, los principales factores de riesgo eran la pobreza - especialmente cuando estaba asociada con el desempleo- y el sexo femenino - por la situación de vulnerabilidad derivada de la mayor inequidad en que se encuentran las mujeres-, seguidos de la inseguridad, posiblemente debido a los recientes conflictos y desastres vividos por la población de la ciudad de El Alto.

Algunos estudios realizados en el marco de la APS en una población de niños y adolescentes en el Distrito Federal, México, indican que casi la mitad de la población estudiada presentaba síntomas de trastornos emocionales y de conducta (13). Asimismo, otras investigaciones realizadas en pacientes de 65 años o más encontraron que los trastornos de ansiedad y depresión son importantes factores asociados con el mal estado de salud y la mala calidad de vida $(14,15)$. En otro estudio se encontró que $60 \%$ de los pacientes con osteoartritis de rodilla y cadera sufría trastornos psiquiátricos, depresión y alexitimia $(16,17)$. La escasa demanda registrada en este estudio, unida al alto porcentaje de médicos que consideraban que no había problemas de salud mental en su área de atención, pueden ser señales del subregistro de los TSMC en esta red de servicios de salud y deben constituir una alerta para la gerencia de la red y otras autoridades sanitarias locales.

En la 55. ${ }^{\mathrm{a}}$ Asamblea Mundial de la Salud, realizada en 2002, la OMS hizo un llamado a trabajar por la salud mental desde los servicios de APS. Según sus proyecciones, la carga mundial de enfermedad por trastornos cerebrales y de salud mental puede aumentar hasta en 15,0\% entre 1990 y 2020 (18). Según datos de 2001, la depresión, la esquizofrenia, el trastorno bipolar, la dependencia de bebidas alcohólicas y la enfermedad de Alzheimer y otras formas de demencia están entre las 13 causas principales de años perdidos por discapacidad en el mundo (1). Estos trastornos también tienen una alta prevalencia en Bolivia (19-21).

En El Alto existe una red social estructurada y organizada, ya sea por juntas vecinales y de manzana o defensorías de la salud, aunque esta no funciona establemente. Los grupos focales manifestaron mucho interés en participar en la solución de los problemas de salud mental que sufren en sus familias, satisfacer las necesidades de atención y lograr una respuesta institucional. Las autoridades municipales, si bien no cuentan con programas específicos para la atención a los TSMC, expresaron su voluntad política para elaborar e implementar esos programas.

En cuanto a la atención de los TSMC por parte del personal sanitario, en las consultas médicas observadas no se identificaron adecuadamente los síntomas y los signos de TSMC y no se diagnosticaron ni se refirieron casos a otros centros. La mayoría de los médicos no conocía de un centro de referencia para estos pacientes o consideraba que eso no era un problema debido a la ausencia de casos. Se observó poca capacidad para el diagnóstico desde un enfoque de la APS, ya que muchos médicos consideraban que los TSMC no constituían un problema en las consultas externas. En este contexto, los trastornos más frecuentemente reconocidos como TSMC por los médicos fueron la depresión y los trastornos por con- 
CUADRO 2. Percepción de las autoridades sanitarias locales y los líderes comunitarios respecto a los trastornos de salud mental y conductuales (TSMC) en los distritos 2 y 3, municipio de El Alto, La Paz, Bolivia, 2004

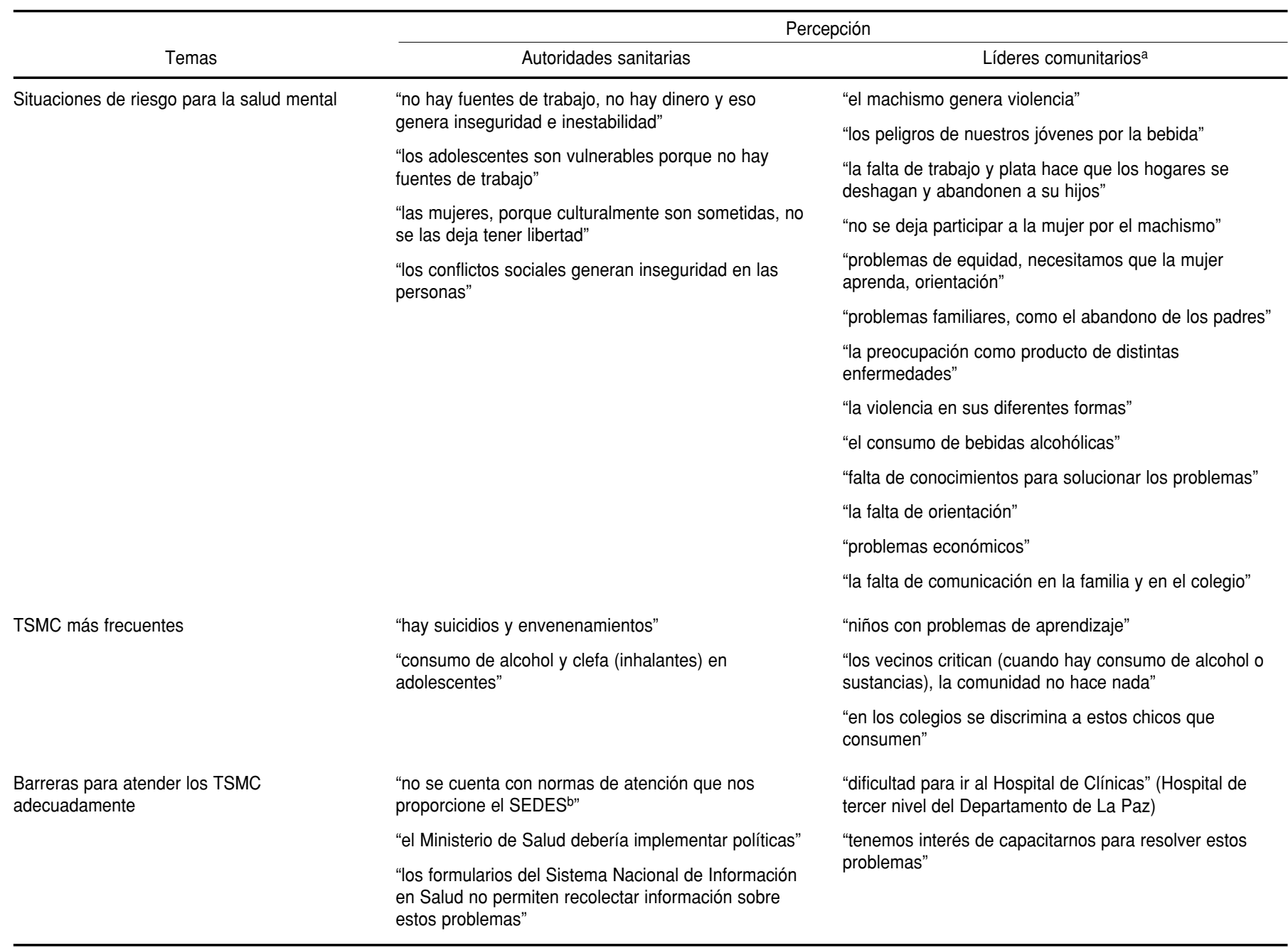

a Representantes de las juntas vecinales y de manzanas y defensorías de la salud.

b SEDES: Servicios Departamentales de Salud, dependencia del Ministerio de Salud y Deportes de Bolivia.

sumo de sustancias psicoactivas, seguidos por la epilepsia y el suicidio.

De esta forma, desde el punto de vista profesional se observaron limitaciones para la atención de los TSMC, causadas por un insuficiente nivel de información y capacitación del personal de salud y la falta de personal especializado. No obstante, la carencia real del tiempo suficiente de consulta para brindar una atención eficaz puede estar desempeñando un importante papel en la situación detectada.

Desde la perspectiva institucional, el ambiente y la infraestructura de la mayoría de los servicios no eran los adecuados para ofrecer una atención apropiada y los únicos medicamentos disponibles en solo algunos de los centros de salud estudiados eran tranquilizantes (diazepam) y anticonvulsivos.

A partir de estos resultados, se puede afirmar que los centros de esta red no están relacionados entre sí y no funcionan como un sistema, sino como unidades aisladas, lo que hace que en muchos de ellos no se conozca la existencia de un servicio especializado en salud mental en el Hospital Corea, como referencia de segundo nivel.

El hecho de no haber encontrado protocolos de atención y programas de prevención, seguimiento y control de pacientes, ni haber observado una estructura de organización y gestión de la asistencia primaria para la salud mental subrayan la necesidad de adoptar un modelo de atención que contenga todos estos elementos.
Los resultados de las entrevistas a los usuarios evidenciaron a su vez la falta de una comunicación efectiva entre el paciente y el médico, que brinde la confianza necesaria al paciente para hacer preguntas, pedir mayor información sobre su enfermedad e indagar acerca de posibles trastornos mentales. Asimismo se constató la ausencia de programas preventivos e informativos dentro de la red analizada. Estos resultados son coherentes con la falta de capacitación y tiempo de consulta, la percepción de los líderes comunitarios y las dificultades de coordinación observadas en la red.

Por último, se debe prestar atención a las recomendaciones hechas por los médicos y los líderes comunitarios para incorporar eficazmente la salud mental 
a los servicios de APS. Entre las recomendaciones más importantes se pueden mencionar la necesidad de establecer un centro de referencia de tercer nivel especializado en salud mental, elevar la capacitación y la formación de los recursos humanos de la red, fortalecer los servicios de la red con instalaciones adecuadas, mejorar el sistema de referencia y contrarreferencia, establecer un modelo de atención para los TSMC con sus correspondientes registros e incorporar profesionales especializados a la red.

Este proceso de diagnóstico participativo y autocrítico de la realidad, las instituciones y las organizaciones de base permitió a los diferentes actores identificar y discutir propuestas para solucionar sus problemas. En este proceso se tomaron en cuenta los criterios de varios actores sociales habitualmente silenciosos - como el personal operativo, la comunidad y los usuarios- en el ámbito de la planificación y la intervención, lo que permitió incorporar elementos tales como la comunicación, la información, la discusión y la búsqueda de consensos.

A partir de estos resultados y las sugerencias propuestas, tanto por el personal sanitario como por los líderes comunitarios, se construyó una propuesta de acción sistematizada, basada en un modelo para redes de servicios de salud, con cuatro componentes: gestión de los servicios, vigilancia epidemiológica, normas y procedimientos de atención, y participación comunitaria. Este modelo se está aplicando actualmente y su comportamiento está en proceso de observación por parte de las autoridades sanitarias locales.

Esta investigación se realizó en un municipio urbano-periférico con características muy particulares, con niveles de pobreza elevados y una fuerte migración rural, por lo que estos resultados y las propuestas participativas desarrolladas deben considerarse solo como un punto de referencia, ya que no reflejan necesariamente la realidad nacional boliviana $o$ de otros países de la Región. No obstante, la situación de la atención en salud mental descrita aquí puede ser común para otros centros de APS del sistema de salud boliviano y podría ser de utilidad extender a todo el sistema la metodología utilizada en el presente estudio.

Dada la baja importancia que se concede a la salud mental en el campo de la salud pública en El Alto, la situación observada se puede agravar por el efecto de otros factores que podrían incrementar la carga de TSMC, como el envejecimiento de la población, el aumento de los problemas sociales y económicos y la agitación social. Es necesario y urgente desarrollar instrumentos que permitan mejorar el diagnóstico y la atención de los TSMC en el marco de una política de Estado.

Se deben fortalecer la formación y la capacitación del personal de los servicios de APS para lograr una adecuada atención de los TSMC. Se debe mejorar la gestión de los servicios y el registro de los trastornos de comportamiento y de salud mental. También se recomienda integrar la participación comunitaria al modelo de abordaje de la salud mental dentro del marco de la APS.

Agradecimientos. Se agradece a Omar Navía y Rubén Colque, de la Facultad de Medicina de la Universidad Mayor de San Andrés, por su colaboración y participación. Un reconocimiento especial a María Elena Peñaranda, del Sustainable Sciences Institute, de los Estados Unidos de América, Oscar Daniel Salomón, del Centro Nacional de Diagnostico e Investigaciones en Endemo-Epidemias, de Argentina, Marco Fidel Suárez, de la OPS/ OMS en Bolivia, y Mercedes Villena, del Instituto Boliviano de la Altura de la UMSA, por sus valiosos aportes en la revisión de este documento.

\section{REFERENCIAS}

1. Organización Mundial de la Salud. Informe sobre la salud en el mundo, 2001. Salud mental: nuevos conocimientos, nuevas esperanzas. Ginebra: OMS; 2001

2. Organización Panamericana de la Salud, Ministerio de Salud y Deportes. Estudio de prevalencia de la violencia doméstica e intrafamiliar en seis municipios: Mizque, Viacha, Riberalta, La Paz, Santa Cruz y Cochabamba. La Paz: OPS; 2003.

3. Nieto M. Psicología para ciencias de la salud: estudio del comportamiento humano ante la enfermedad. Madrid: McGraw-Hill Interamericana; 2004.

4. Oblitas LA. Psicología de la salud y calidad de vida. México: Fuentes I; 2006.

5. Caja Nacional de Salud. Estadísticas de psiquiatría infantil. La Paz: CNS; 2002.

6. República de Bolivia, Ministerio de Salud y Previsión Social. Conclusiones y recomendaciones del taller para la elaboración de una propuesta para el Plan Nacional de Salud Mental, 2003-2004. La Paz: OPS; 2003.

7. Servicio Departamental de Salud, Prefectura del Departamento de La Paz. Estadísticas de salud, 2003. La Paz: Servicio Nacional de Información en Salud; 2003. (Anuario No. 17).

8. Instituto Nacional de Estadística. Atlas estadístico de municipios, 2005. La Paz; Pro- grama de las Naciones Unidas para el Desarrollo; 2005.

9. Instituto Nacional de Estadística. Situación de indicadores básicos, 2003. La Paz: Ministerio de Salud y Deportes; 2003.

10. Gaceta Oficial de Bolivia. Decreto Supremo No. 26875: modelo de gestión y directorio local de salud. La Paz: Presidencia de la República; 2002.

11. Instituto Nacional de Estadística. Censo Nacional de Población y Vivienda 2001. La Paz: INE; 2002.

12. Bru Martín P, Basagoiti M. La investigaciónacción participativa como metodología de mediación e integración socio-comunitario. Madrid: McGraw-Hill Interamericana; 2003.

13. Caraveo-Anduaga JJ, Colmenares-Bermúdez E, Martínez-Vélez NA. Síntomas, percepción y demanda de atención en salud mental en niños y adolescentes de la ciudad de México. Salud Publica Mex. 2002:44:492-8.

14. Vicente B, Kohn R, Saldivia S, Rioseco P, Torres $S$. Patrones de uso de servicios entre adultos con problemas de salud mental en Chile. Rev Panam Salud Publica. 2005;18(4-5):263-70.

15. Federación Mundial para la Salud Mental. Efectos del trauma y la violencia sobre niños y adolescentes. Washington, D.C.: FMSM, OMS; 2002.
16. Asociación Estadounidense de Psiquiatría Manual diagnóstico y estadístico de los trastornos mentales. Barcelona: Masson; 2002.

17. Organización Mundial de la Salud. Trastornos mentales y del comportamiento. Washington, D.C.: OPS; 1995. (Publicación Científica No. 554.)

18. Organización Mundial de la Salud. Informe de la 55. ${ }^{\text {a }}$ Asamblea Mundial de la Salud. Ginebra: OMS; 2002

19. República de Bolivia, Ministerio de Salud y Deportes, Dirección Nacional de Desarrollo de Servicios de Salud. Programa Nacional de Salud Integral de los y las Adolescentes. Plan Nacional para la Salud y el Desarrollo Integral de los y las Adolescentes, 2004-2008. La Paz: OPS; 2004.

20. República de Bolivia, Ministerio de Salud y Deportes. Alcohol. La Paz: OPS; 2004.

21. República de Bolivia, Ministerio de Salud y Deportes. Programa Nacional de Salud Mental, Prevención y Rehabilitación. Trastornos emocionales y conductuales de niños y adolescentes. La Paz: MSD; 2003.

Manuscrito recibido el 6 de noviembre de 2007. Aceptado para publicación, tras revisión, el 14 de agosto de 2008. 
ABSTRACT Objectives. To determine the mental health and behavioral disorders (MHBD) treated by a Bolivian health network; and to gauge the perspective of the health professionals, community members, and care-providers in order to identify ways to bet-

The state of mental health primary care in the social services of El Alto, La Paz, Bolivia

Key words Mental health, health services, primary health care, Bolivia.
Methods. A descriptive cross-sectional study was conducted from January to December 2005 in 15 public health centers in districts 2 and 3 of the Corea Network, one of four urban networks in the municipality of El Alto, province of Murillo, department of La Paz, Bolivia. The research and active participation methodology was employed through interviews, focus group sessions, review of external consultation logs and clinical histories, and active observation.

Results. Only $0.05 \%$ of primary care visits were for MHBD. Among the barriers identified by doctors were: lack of a specialized reference center $(60.0 \%)$, not enough time $(40.0 \%)$, a shortage of specialized personnel (33.3\%), the drug supply (20.0\%), and undertrained staff $(16.6 \%)$. The departmental and federal programs have limited human resources training, standards of care are nonexistent, and the information system does not collect data on MHBD. Community members amply identified the mental health problems and care needs that, to date, have not been met by the social services.

Conclusiones. Training and development of primary care services staff must be expanded so as to provide the proper care for mental health disorders. Improvements must be made in the management of health care services and the reporting of behavioral and mental health disorders. Another recommendation is to incorporate community participation in the model approach to mental health within the framework of primary health care.

\section{GOINGgINTERATIONAL On course for your career! Course Catalogue "medicine \& health" 2009 Complete Edition \\ Researched and compiled for you: 2500 top courses and programmes in abstract form ... for easy comparison and orientation 750 organisers and institutions of higher education worldwide Valid from October 2008 until July 2010

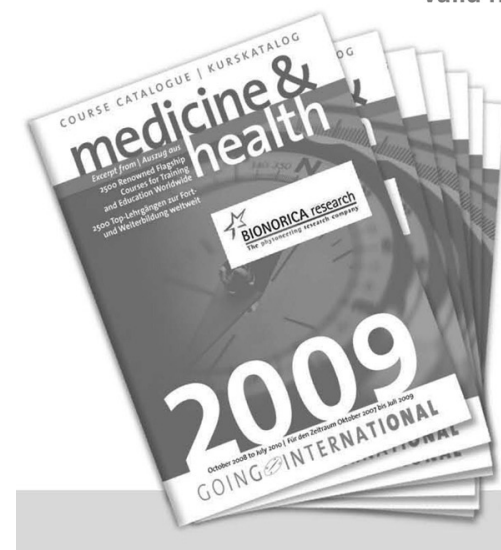 Price: $€ 37.15^{*}$ \\ Thematic special editions \\ New: Thematic editions of "medicine \& health 2009 " presenting courses, master programmes and conferences \\ Special Editions Topics: I) Courses for Hospital Management and Information Technology . II) Training for Humanitarian Assistance and Complex Emergencies - III) Courses in International Health and Development Cooperation - IV) Courses in Interdisciplinary Medicine . V) Courses for Medical Specialists - VI) Programmes in Public Health. VII) Courses for Researchers, training in Evidence-Based Decision-Making Prices from $€ 9.90$ to $€ 12.10^{*}$}

